hep-th/0204143

\title{
Field Theory of Tachyon Matter
}

\author{
Ashoke Sen \\ Harish-Chandra Research Institute \\ Chhatnag Road, Jhusi, Allahabad 211019, INDIA \\ and \\ Department of Physics, Penn State University \\ University Park, PA 16802, USA \\ E-mail: asen@thwgs.cern.ch, sen@mri.ernet.in
}

\begin{abstract}
We propose a field theory for describing the tachyon on a brane-antibrane system near the minimum of the potential. This field theory realizes two known properties of the tachyon effective action: 1) absence of plane-wave solutions around the minimum, and 2) exponential fall off of the pressure at late time as the tachyon field evolves from any spatially homogeneous initial configuration towards the minimum of the potential. Classical solutions in this field theory include non-relativistic matter with arbitrary spatial distribution of energy.
\end{abstract}


Dynamics of the tachyon on D-brane anti-D-brane system or unstable D-branes in superstring theory and bosonic string theory have been investigated during the last few years. Of the various known properties of the classical tachyon effective action, 1 two specific properties which specify the behaviour of the tachyon effective action around the minimum of the potential are as follows:

1. Since the minimum of the potential describes a configuration where there are no D-branes [1], around this minimum there are no physical open string excitations. Translated to a property of the tree level tachyon effective action, this implies that there are no plane wave solutions to the linearized equations of motion around the minimum of the tachyon potential.

2. If we let the tachyon roll beginning with any spatially homogeneous initial configuration [2], it evolves asymptotically towards its minimum instead of oscillating about the minimum[3], [1]. The total energy density is conserved during the evolution but the pressure evolves to zero. The information about the tachyon effective action near the minimum of the potential is encoded in the late time behaviour of the pressure. This is given by 3 , 田

$$
p=-K e^{-\alpha x^{0}}
$$

where $K$ is an irrelevant normalization constant which can be changed by shifting the origin of the time coordinate $x^{0}$, and $\alpha$ is given (in the $\alpha^{\prime}=1$ unit) by

$$
\begin{array}{rlr}
\alpha & =1 \quad \text { for bosonic string theory } \\
& =\sqrt{2} \quad \text { for superstring theory. }
\end{array}
$$

Several field theory models have been proposed for realising the first property. These models are of two kind.] In the first kind[7] the tachyon potential is non-singular at the minimum of the potential but the absence of physical plane-wave solutions has its origin in the structure of the kinetic term (which typically involves higher derivative terms). This

\footnotetext{
${ }^{1}$ Although we shall use the language of tachyon effective action obtained by integrating out the other open string modes, we should note that many of these results are derived using the technology of full string field theory, containing all the infinite number of fields describing the dynamics of the open string.

${ }^{2}$ Since we are interested in studying tree level properties of the tachyon potential, we shall ignore the backreaction of gravity and other closed string fields on the evolution of the tachyon. Once the form of the action is derived by this method, one can of course couple it to background gravitational field and study the evolution of the coupled system. These effects have been studied in refs. [5]. For some earlier attempts at the study of time dependent solutions for the tachyon field see [6].

${ }^{3}$ Here we are stating the results in terms of properly redefined field $\phi$ for which the two derivative term has the standard form $-\frac{1}{2} \eta^{\mu \nu} \partial_{\mu} \phi \partial_{\nu} \phi$.
} 
possibility seems to be realized[8] in the explicit analysis of the string field theory based on $*$-product interaction [9]. In the second kind of field theory models [10] the tachyon potential is singular at the minimum. In particular the second derivative of the potential blows up and hence the tachyon mass becomes infinite. Analysis based on boundary string field theory 11] seems to support this view 12]. Of course these results are not mutually incompatible, since an effective field theory of the first kind may be turned into one of second kind and vice versa by a suitable field redefinition that involves (infinite number of) derivatives of the tachyon field.

A field theory model that realises the evolution of the system to a pressureless gas was proposed in [4] following earlier proposals in [13, 14]. The action was taken to be

$$
S=-\int d^{p+1} x V(T) \sqrt{-\operatorname{det} A}
$$

where

$$
A_{\mu \nu}=\eta_{\mu \nu}+\partial_{\mu} T \partial_{\nu} T
$$

However in this proposal the tachyon potential $V(T)$ was left undetermined. The only property of $V(T)$ that was determined is that the minimum of $V(T)$ where $V(T)=0$ should be at $T=\infty$.

In this paper we shall show that by taking $V(T) \propto e^{-\alpha T / 2}$ for large $T$ in the action (3), we can satisfy both the requirements given above: the absence of plane-wave solutions around the tachyon vacuum, and the exponential fall off of the pressure for large $x^{0}$. The constant of proportionality can be absorbed into a constant shift of $T$. Thus the proposed tachyon effective action for large $T$ on an unstable D-p-brane system is

$$
S=-\int d^{p+1} x e^{-\alpha T / 2} \sqrt{-\operatorname{det} A}=-\int d^{p+1} x e^{-\alpha T / 2} \sqrt{1+\eta^{\mu \nu} \partial_{\mu} T \partial_{\nu} T},
$$

where $A_{\mu \nu}$ is given by eq.(41). We expect this to be a valid approximation when the second and higher order derivatives of $T$ are small. As we shall see, the tachyon matter does satisfy this condition.

On a brane-antibrane system where $T$ is a complex field, the tachyon potential is given by $e^{-\alpha|T| / 2}$. In this case the action (5) can be regarded as the restriction of the full action to the case $\Im(T)=0$. We shall carry out our analysis for this restricted field configuration. The imaginary part of the tachyon, which can be regarded as the Goldstone mode associated to the broken $U(1)$ phase symmetry, is absorbed by the $\mathrm{U}(1)$ gauge field due to Higgs mechanism, and is expected to decouple from the dynamics near the minimum of the potential.

We shall first verify that the action (5) produces the correct large $x^{0}$ behaviour of the pressure. For this analysis we can restrict to spatially homogeneous, time dependent 
field configurations. As discussed in [14, 4], for such configurations the conserved energy density is given by:

$$
T_{00}=e^{-\alpha T / 2}\left(1-\left(\partial_{0} T\right)^{2}\right)^{-1 / 2} .
$$

Since $T_{00}$ is conserved, we see that for any given $T_{00}$, as $T \rightarrow \infty, \partial_{0} T \rightarrow 1$. In particular, for large $x^{0}$ the solution has the form:

$$
T=x^{0}+C e^{-\alpha x^{0}}+\mathcal{O}\left(e^{-2 \alpha x^{0}}\right) \text {. }
$$

In order to see that (17) gives the correct form of the solution we simply need to note that the leading contribution to $T_{00}$ computed from this configuration remains constant in time:

$$
T_{00} \simeq \frac{1}{\sqrt{2 \alpha C}}
$$

The pressure associated with this configuration is given by 14 , 4 :

$$
p=-e^{-\alpha T / 2}\left(1-\left(\partial_{0} T\right)^{2}\right)^{1 / 2} \simeq-\sqrt{2 \alpha C} e^{-\alpha x^{0}} .
$$

This is in precise agreement with (1).

Next we shall demonstrate the absence of plane-wave solutions. First let us expand the action in powers of derivatives of $T$ up to terms containing at most two powers of derivatives. This gives

$$
S=-\int d^{p+1} x e^{-\alpha T / 2}\left(1+\frac{1}{2} \eta^{\mu \nu} \partial_{\mu} T \partial_{\nu} T+\ldots\right)
$$

where ... denotes terms with higher powers of derivatives. Defining

$$
\phi=e^{-\alpha T / 4},
$$

we get

$$
S=\int d^{p+1} x \frac{16}{\alpha^{2}}\left(-\frac{1}{2} \eta^{\mu \nu} \partial_{\mu} \phi \partial_{\nu} \phi-\frac{\alpha^{2}}{16} \phi^{2}+\ldots\right) .
$$

The minimum of the potential is at $\phi=0$. If we ignore the higher derivative terms then the quantization of the action seems to lead to a scalar particle of mass $\alpha / 2 \sqrt{2}$. This is reflected in the fact that the equations of motion without the higher derivative terms contain a plane-wave solution of the form:

$$
\phi=a e^{i k_{\mu} x^{\mu}},
$$

\footnotetext{
${ }^{4}$ Note that since for this configuration $\partial_{0}^{n} T$ for $n>1$ falls off exponentially, the contribution from possible higher derivative corrections like $\left(\partial_{\mu} \partial^{\mu} T\right)^{2}$ inside the square root on the right hand side of (5) will be suppressed.
} 
with $-\eta^{\mu \nu} k_{\mu} k_{\nu}=\alpha^{2} / 8$ and $a$ an arbitrary constant.

We shall now show that when we take into account the effect of the higher derivative terms, (13) ceases to be a solution of the equations of motion for any $k_{\mu}$. 9 To show this we note that the full action (5) written in terms of $\phi$ defined in (11) takes the form:

$$
S=-\int d^{p+1} x \phi^{2} \sqrt{1+\frac{16}{\alpha^{2}} \phi^{-2} \eta^{\mu \nu} \partial_{\mu} \phi \partial_{\nu} \phi}
$$

The action is homogeneous of degree 2 in $\phi$. The equations of motion derived from this action is given by:

$$
-\eta^{\mu \nu} \partial_{\mu}\left(\frac{\partial_{\nu} \phi}{\sqrt{1+\frac{16}{\alpha^{2}} \phi^{-2} \eta^{\mu \nu} \partial_{\mu} \phi \partial_{\nu} \phi}}\right)+\frac{\alpha^{2}}{8} \frac{\phi+\frac{8}{\alpha^{2}} \phi^{-1} \eta^{\mu \nu} \partial_{\mu} \phi \partial_{\nu} \phi}{\sqrt{1+\frac{16}{\alpha^{2}} \phi^{-2} \eta^{\mu \nu} \partial_{\mu} \phi \partial_{\nu} \phi}}=0 .
$$

Substituting (13) into (15) we get:

$$
\frac{a}{\sqrt{1-\frac{16}{\alpha^{2}} \eta^{\mu \nu} k_{\mu} k_{\nu}}}=0
$$

Clearly this equation has no non-trivial solution for finite values of $k_{\mu}$. This establishes the absence of plane-wave solutions. (Note that if we expand the left hand side of this equation in powers of $k_{\mu}$ and keep up to quadratic terms, we reproduce the mass-shell condition $-\eta^{\mu \nu} k_{\mu} k_{\nu}=\alpha^{2} / 8$ derived earlier.)

Absence of plane wave solutions does not imply absence of other classical solutions however. We have already seen the existence of solutions with constant (but arbitrary) energy density. As emphasized in [14] (see also [15, 16]) the correct excitations of the system can be found by working in the hamiltonian formalism. Defining the momentum conjugate to $T$ as:

$$
\Pi(x)=\frac{\delta S}{\delta\left(\partial_{0} T(x)\right)},
$$

we can construct the Hamiltonian $H$ following [14]:

$$
H=\int d^{p} x \mathcal{H}, \quad \mathcal{H}=T_{00}=\sqrt{\Pi^{2}+e^{-\alpha T}} \sqrt{1+\partial_{i} T \partial_{i} T} .
$$

\footnotetext{
${ }^{5}$ Note that (13) describes a complex solution. Since the full equations of motion are homogeneous of degree one, but not linear, even if we had a solution of the form (13), it is not clear how we could construct a real solution since we cannot superpose the solutions. We shall show however that even this complex solution does not exist.

${ }^{6}$ Note again that since for the configuration (13) $\partial_{\mu} \partial_{\nu} T$ vanishes, contribution to the equations of motion from possible higher derivative corrections like $\left(\partial_{\mu} \partial^{\mu} T\right)^{2}$ inside the square root on the right hand size of (5) vanishes.
} 
For large $T$ we can ignore the $e^{-\alpha T}$ term, and the equations of motion take the form:

$$
\begin{gathered}
\partial_{0} \Pi(x)=-\frac{\delta H}{\delta T(x)}=\partial_{j}\left(\Pi(x) \frac{\partial_{j} T}{\sqrt{1+\partial_{i} T \partial_{i} T}}\right) . \\
\partial_{0} T(x)=\frac{\delta H}{\delta \Pi(x)}=\sqrt{1+\partial_{i} T \partial_{i} T}
\end{gathered}
$$

for $\Pi(x)>0$. Thus we can get a solution to the equations of motion by taking $\partial_{i} T=$ $\partial_{0} \Pi=0, \partial_{0} T=1$. This gives

$$
\Pi(x)=f(\vec{x}), \quad T(x)=x^{0},
$$

where $f(\vec{x})$ is any arbitrary function of the spatial coordinates. The energy density associated with such a solution is proportinal to $f(\vec{x})$. This shows that the system admits classical solutions with energy density which is time independent but has arbitrary dependence on the spatial coordinates. Classically this energy density can be as low as we like. The ability to create configurations with arbitrarily low energy density can be traced to the scale invariance:

$$
T(x) \rightarrow \lambda^{-1} T(\lambda x), \quad \Pi(x) \rightarrow \lambda^{p+1} \Pi(\lambda x),
$$

under which the Poisson brackets and the equations of motion remain unchanged, and $\mathcal{H}(x) \rightarrow \lambda^{p+1} \mathcal{H}(\lambda x)$.

Since the original theory is Lorentz invariant, it is clear that given a localized density of tachyon matter we should be able to boost it to an arbitrary velocity. Intuitively we also expect that is should be possible to construct configurations with different local velocities at different points. This can be seen to be the case by rewriting the equations of motion in a slightly different form. We define:

$$
u_{\mu}=\partial_{\mu} T, \quad \epsilon(x)=\Pi(x) / \partial_{0} T(x) .
$$

The equations of motion then take the suggestive form:

$$
\eta^{\mu \nu} u_{\mu} u_{\nu}=-1, \quad \partial_{\mu}\left(\epsilon(x) u^{\mu}\right)=0
$$

The energy momentum tensor can be computed following [14. Expressed in terms of these new variables, $T_{\mu \nu}$ take the form:

$$
T_{\mu \nu}=\epsilon(x) u_{\mu} u_{\nu}
$$

These are precisely the equations govering gradient flow of non-interacting dust, with $u_{\mu}$ interpreted as the local velocity vector. These equations are expected to be valid as long as $\partial_{\mu} u_{\nu}$ is small in magnitude (in string units). 
Although in our analysis we have ignored the coupling of the tachyon to various massless fields on the D-brane world-volume, these can be easily incorporated following [17, 13, 14, 15]. We can generalize (5) to curved background space-time by replacing $\eta_{\mu \nu}$ in eq.(化) by the closed string metric $g_{\mu \nu}$. If we also have anti-symmetric tensor field background then $\eta_{\mu \nu}$ should be replaced by $g_{\mu \nu}+B_{\mu \nu}$. The dilaton $\phi$ couples via an overall factor of $e^{-\phi}$ multiplying the lagrangian density. For non-BPS D-p-brane of type II string theories, another quantity of interest is the coupling of the tachyon to Ramond-Ramond (RR) field $C^{(p)}$. In particular it is known [18] that the D-p-brane world-volume theory has a coupling:

$$
\int d^{p+1} x f(T) d T \wedge C^{(p)}
$$

where $f(T)$ is some function of the tachyon field $T$. We can determine the behaviour of $f(T)$ for large $T$ if we can find the source of the RR field that is generated by the rolling tachyon field [2]. This can be computed from the boundary state associated with the solution. Although we have not performed a systematic analysis of the problem along the lines of [3, 团, general arguments based on symmetry and other considerations lead us to guess that (in $\alpha^{\prime}=1$ unit) the source for the $\mathrm{RR} p$-form field is proportional to:

$$
\sin (\widetilde{\lambda} \pi)\left[\frac{e^{x^{0} / \sqrt{2}}}{1+\sin ^{2}(\widetilde{\lambda} \pi) e^{\sqrt{2} x^{0}}}-\frac{e^{-x^{0} / \sqrt{2}}}{1+\sin ^{2}(\widetilde{\lambda} \pi) e^{-\sqrt{2} x^{0}}}\right],
$$

where $\tilde{\lambda}$ is the parameter labelling the total energy density of the system:

$$
T_{00}=\frac{\mathcal{T}_{p}}{2}(1+\cos (2 \widetilde{\lambda} \pi)),
$$

$\mathcal{T}_{p}$ being the tension of the non-BPS D-p-brane. Thus for large $x^{0}$ the source is proportional to $e^{-x^{0} / \sqrt{2}}$. Since $T \simeq x^{0}$ for large $x^{0}$, this indicates that for large $T$, the coupling of the RR $p$-form field to the tachyon has the form:

$$
\int d^{p+1} x e^{-T / \sqrt{2}} d T \wedge C^{(p)},
$$

up to an overall normalization constant.

There are many issues which call for further investigation. As we have seen, the effective field theory describing tachyon matter has classical solutions with localized energy densities. It will be interesting to construct the two dimensional conformal field theories associated with these solutions along the lines of refs. 3, [1 where the conformal field theories associated with spatially homogeneous energy densities were constructed. (Of course, one possibility is to take an arbitrary spatial distribution of coincident D0-D̄0 pair and construct the rolling tachyon solution on each such pair.) Another issue of importance is the effect of quantum corrections on such background. 
Acknowledgement: I would like to thank L. Kofman, J. Maldacena and T. Padmanabhan for useful discussions. This work was supported in part by a grant from the Eberly College of Science of the Penn State University.

\section{References}

[1] A. Sen, JHEP 9808, 010 (1998) arXiv:hep-th/9805019; JHEP 9808, 012 (1998) arXiv:hep-th/9805170; JHEP 9912, 027 (1999) arXiv:hep-th/9911116.

[2] M. Gutperle and A. Strominger, "Spacelike branes," arXiv:hep-th/0202210.

[3] A. Sen, "Rolling Tachyon," arXiv:hep-th/0203211.

[4] A. Sen, "Tachyon matter," arXiv:hep-th/0203265.

[5] G. W. Gibbons, arXiv:hep-th/0204008; M. Fairbairn and M. H. Tytgat, arXiv:hepth/0204070; S. Mukohyama, arXiv:hep-th/0204084; A. Feinstein, arXiv:hepth/0204140; T. Padmanabhan, arXiv:hep-th/0204150; A. Frolov, L. Kofman and A. A. Starobinsky, arXiv:hep-th/0204187; D. Choudhury, D. Ghoshal, D. P. Jatkar and S. Panda, arXiv:hep-th/0204204; X. Li, J. Hao and D. Liu, arXiv:hepth/0204252; G. Shiu and I. Wasserman, arXiv:hep-th/0205003.

[6] C. Acatrinei and C. Sochichiu, arXiv:hep-th/0104263; S. H. Alexander, Phys. Rev. D 65, 023507 (2002) arXiv:hep-th/0105032; A. Mazumdar, S. Panda and A. PerezLorenzana, Nucl. Phys. B 614, 101 (2001) arXiv:hep-ph/0107058; S. Sarangi and S. H. Tye, arXiv:hep-th/0204074.

[7] L. Brekke, P. G. Freund, M. Olson and E. Witten, Nucl. Phys. B 302, 365 (1988); P. H. Frampton and Y. Okada, Phys. Rev. D 37, 3077 (1988); D. Ghoshal and A. Sen, Nucl. Phys. B 584, 300 (2000) arXiv:hep-th/0003278; J. A. Minahan, JHEP 0103, 028 (2001) arXiv:hep-th/0102071.

[8] V. A. Kostelecky and S. Samuel, Nucl. Phys. B 336, 263 (1990); A. Sen and B. Zwiebach, JHEP 0010, 009 (2000) arXiv:hep-th/0007153; W. Taylor, JHEP 0008, 038 (2000) arXiv:hep-th/0008033; H. Hata and S. Teraguchi, JHEP 0105, 045 (2001) arXiv:hep-th/0101162]; I. Ellwood and W. Taylor, Phys. Lett. B 512, 181 (2001) arXiv:hep-th/0103085; L. Rastelli, A. Sen and B. Zwiebach, arXiv:hepth/0012251.

[9] E. Witten, Nucl. Phys. B 268, 253 (1986). N. Berkovits, Nucl. Phys. B 450, 90 (1995) [Erratum-ibid. B 459, 439 (1995)] arXiv:hep-th/9503099]. 
[10] J. A. Minahan and B. Zwiebach, JHEP 0009, 029 (2000) arXiv:hep-th/0008231; JHEP 0103, 038 (2001) arXiv:hep-th/0009246; JHEP 0102, 034 (2001) arXiv:hepth/0011226.

[11] E. Witten, Phys. Rev. D 46, 5467 (1992) arXiv:hep-th/9208027; Phys. Rev. D 47, 3405 (1993) arXiv:hep-th/9210065; S. L. Shatashvili, Phys. Lett. B 311, 83 (1993) arXiv:hep-th/9303143.

[12] A. A. Gerasimov and S. L. Shatashvili, JHEP 0010, 034 (2000) arXiv:hepth/0009103]; D. Kutasov, M. Marino and G. W. Moore, JHEP 0010, 045 (2000) arXiv:hep-th/0009148]; arXiv:hep-th/0010108.

[13] M. R. Garousi, Nucl. Phys. B 584, 284 (2000) arXiv:hep-th/0003122; E. A. Bergshoeff, M. de Roo, T. C. de Wit, E. Eyras and S. Panda, JHEP 0005, 009 (2000) arXiv:hep-th/0003221; J. Kluson, Phys. Rev. D 62, 126003 (2000) arXiv:hep-th/0004106.

[14] G. W. Gibbons, K. Hori and P. Yi, "String fluid from unstable D-branes," Nucl. Phys. B 596, 136 (2001) arXiv:hep-th/0009061.

[15] O. Bergman, K. Hori and P. Yi, "Confinement on the brane," Nucl. Phys. B 580, 289 (2000) arXiv:hep-th/0002223; J. A. Harvey, P. Kraus and F. Larsen, JHEP 0012, 024 (2000) arXiv:hep-th/0010060]; F. Larsen, Int. J. Mod. Phys. A 16, 650 (2001) arXiv:hep-th/0010181.

[16] U. Lindstrom and R. von Unge, Phys. Lett. B 403, 233 (1997) arXiv:hepth/9704051; H. Gustafsson and U. Lindstrom, Phys. Lett. B 440, 43 (1998) arXiv:hep-th/9807064; U. Lindstrom, M. Zabzine and A. Zheltukhin, JHEP 9912, 016 (1999) [arXiv:hep-th/9910159].

[17] A. Sen, JHEP 9910, 008 (1999) arXiv:hep-th/9909062; J. Math. Phys. 42, 2844 (2001) arXiv:hep-th/0010240.

[18] E. Witten, JHEP 9812, 019 (1998) arXiv:hep-th/9810188; P. Horava, Adv. Theor. Math. Phys. 2, 1373 (1999) arXiv:hep-th/9812135; A. Sen, arXiv:hep-th/9904207. 